\title{
Research on Image Processing in Weld Seam Tracking with Laser Structured Light
}

\author{
Xiwen Liư ${ }^{1, a}$, Chaoying Liü,b \\ ${ }^{1}$ Department of Electronic Information \& Electrical Engineering, Zhaoqing University,Zhaoqing, \\ Guangdong 526061, China \\ 2Zhaoqing GYK General Aviation Academy, Zhaoqing, Guangdong 526070,China \\ aliuxiwen1111@163.com, ${ }^{\mathrm{b}}$ chaoying1221@126.com
}

Keywords: weld seam tracking; laser structured light; Radon transform; improved FCM.

Abstract. According to the image characteristics in weld seam tracking with laser vision, Radon transform is first used to find the location of the laser stripe; then, the transformed image is processed by using the way that the pixel information caused by laser stripe is reserved and the other information by noises is removed, and then inverse Radon transform is applied for image recovery. Finally, improved fast FCM clustering is used for image segmentation so that the groove area can be recognized. These methods can bring good effects with little time cost, and the antidisturbance performance is well, therefore they are suitable for weld seam tracking with laser vision.

\section{Introduction}

Auto-welding will be widely used in the future, and weld seam tracking is one of the key technologies in auto-welding. At present, vision sensing method is always applied for weld seam tracking. For the sake of much noises from voltaic arc and molten steel spray, it is difficult to get good weld seam image using common optical sources[1]. Laser has two advantages in comparison with common optical sources[2], one is that it has concentrative energy, the other is that it can't be easily affected by environment. Therefore, laser can be used as optical sources for weld seam tracking. As large laser generator is too expensive, small single-stripe laser generator is often used for weld seam tracking today. Though the weld seam image with single-stripe laser is relatively good, it can't fully avoid noises from voltaic arc, molten steel spray and smog. In order to obtain the weld groove zone precisely, it is necessary to eliminate these noises.

According to the characteristics of single-stripe laser, in this paper, Radon transform and fuzzy $\mathrm{C}$-means clustering segmentation are applied for image processing. Using these methods, the laser stripe can be detected precisely, the noises in weld seam image can be elimated effectively, and the weld seam groove zone can be recognized easily.

\section{Theory of Radon Transform.}

In two-dimensional space, $(x, y)$ represents the point coordinate, and $f(x, y)$ is a function in $R^{2}$ field, if $L$ is an arbitrary line in two-demensional space, then the integral of $f(x, y)$ along line $L$ is

$$
p=\int_{L} f(x, y) d l
$$

As shown in Fig.1, $\theta$ represents the inclination of Line $L$, rotate xoy coordinate system to make its y-axis to be parallel to Line $L$, and the new coordinate system is called tos, therefore:

$$
\left\{\begin{array}{l}
x=t \cos \theta-s \sin \theta \\
y=t \sin \theta+s \cos \theta
\end{array}\right.
$$




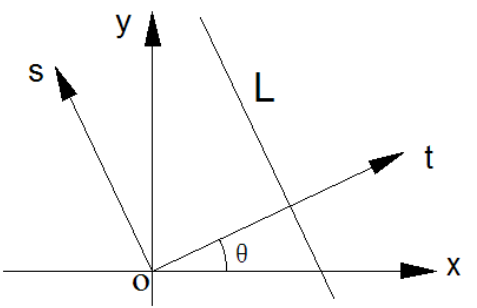

Fig.1 Two-dimensional Radon transform

The integral of $f(x, y)$ along line $L$ can also be expressed as:

$$
p(\theta, t)=\int_{-\infty}^{+\infty} f((t \cos \theta-s \sin \theta),(t \sin \theta+s \cos \theta)) d s
$$

The above formula is the definition of continuous Radon transform, it can be seen that the line integrals of function $f(x, y)$ also can be looked as sum of pixels' projection to $t$ axis. For digital signals, discrete Radon transform should be used. Therefore, the Radon transform of twodimensional image along one line is the sum of pixels which locate in the line. Obviously, if a line exists in the image, this line must be corresponding to the extreme value of $p(\theta, t)$ after Radon transform. Therefore, being similar to Hough transform, Radon transform can be used for line detecting.

Radon transform has its inverse transform, when an image Radon transform is finished, it can be recovered precisely by inverse Radon transform. Radon transform and its reverse transform both have rapid realization methods.

\section{Characteristics of image with line after Radon transform}

As shown in Fig.2a, there is a $192 \times 192$ size image which contains a vertical line. As shown in Fig.2b, a coordinate system is build up, the coordinate system's original point is set as the center point of the image, and its $\mathrm{X}$-axis is along horizontal direction. Discrete Radon transform is applied to Fig.2a. As the inclination angle cycle of $p(\theta, t)$ is $180^{\circ}$, therefore $\theta \in\left[0^{\circ}, 179^{\circ}\right]$, and the angle $\theta$ is taken as integers. If $\theta$ varies, the effective length of $t$ also varies, suppose $t_{\theta}$ is the effective projection length, then

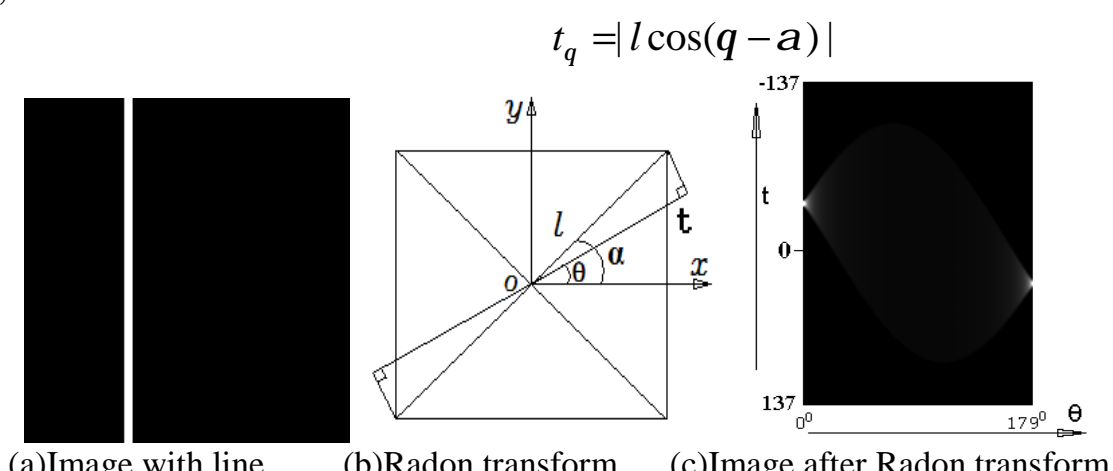

(a)Image with line

(b)Radon transform

(c)Image after Radon transform

Fig.2 Radon transform of image with line

In (4), $l$ represents the image's diagonal line, $\alpha$ is the included angle between the image's diagonal line and horizontal line, obviously, the effective projection length $t_{\alpha}$ is maximal when $\theta$ equals to $\alpha$. In order to save computing time and make the projection zone symmetry about $t$ axis, the actual maximal projection length $t_{\alpha}^{\prime}$ should be made odd and a little lager than the diagonal length $l$. For a $192 \times 192$ size image, the maximal effective projection length can be calculated as 275 which is a little larger than $192 \sqrt{2}$. If $p(t, \theta)$ is shown as a image, a $180 \times 275$ size image is 
gotten, as shown in Fig.2c, the x-axis represents angle $\theta$, and the $y$-axis represents distance $t$, if $|t|>\frac{1}{2} t_{\theta}$, then $p(\theta, t)=0$.

In Fig.2c, the brightest point corresponds to the vertical line in Fig.2a, and its x-axis coordinate is $0^{0}$. Therefore, the vertical line's location and angle in Fig.2a can be decided from the brightest point's x-y coordinates in Fig.2c. Additionally, the line information mainly concentrates nearby the brightest point, and it is symmetrical about the image center.

\section{Characteristics of Weld Seam Image with Laser Vision}

In the procedure of weld seam tracking, the location and angle of laser emitter and camera are often adjusted for getting vertical laser stripe image which can be easily processed by computer. Additionally, in order to avoid the disturbance of voltaic arc and prevent the harm of high temperature, the laser emitter and camera are often placed $25 \mathrm{~cm}$ before the welding torch, but the image obtained can still be polluted by molten steel spray, arc, rust, greasy dirt, etc. These can severely affect the recognition of weld seam zone. Fig.3 shows a typical V shape groove weld seam image, it can be seen that some spray noises are much brighter than the laser stripe, these noises must be cleared in order to recognize the weld seam zone precisely.

In the weld seam image with laser vision, the laser stripe is mainly made up of vertical lines. Therefore, when Radon transform is applied to the weld seam image, the information of the laser stripe mainly concentrates nearby the corresponding extreme point, so image processing can be made in Radon transform domain to eliminate the noises.

\section{Image Processing with Laser Vision}

Radon transform. Using Radon transform to Fig.3, $\theta \in\left[0^{0}, 179^{\circ}\right]$, as shown in Fig.4, the image after Radon transform is gotten.

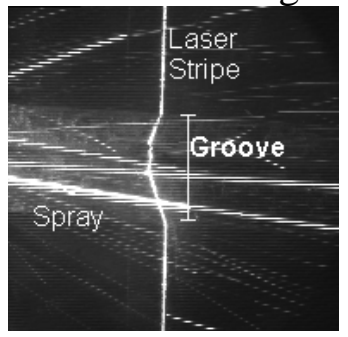

Fig.3 Weld seam Image

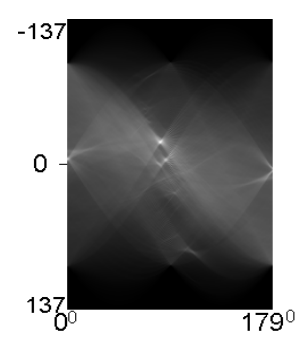

Fig.4 Image after Radon transforming

From Fig.4, it can be seen that the brightest point's $\mathrm{x}$-axis coordinate is about $80^{\circ}$, this point corresponds to the noises of original image; as the laser stripe in original image is almost vertical, while the probability of noises being vertical is very little. After Radon transform, the extreme point corresponds to the laser stripe in original image should be nearby $0^{0}$ or $179^{0}$. Therefore, in Fig. 4 , coordinates of the point correspond to the laser stripe are $\left(1^{0},-4\right)$, this point is also called singular point, through the singular point's coordinates, location and angle of the laser stripe can be gotten; additionally, if the pixels closed to this point are enhanced, and the other pixels are weakened, the noises will be eliminated effectively after recovery.

Image processing in Radon domain. The processing methods are as follows:

Step 1: Select a suitable rectangular area whose center is the singular point corresponding to the laser stripe. By experiment, that the half width of the rectangle is taken as 20 is appropriate.

Step 2: As shown in Fig.5a, keep pixels in the rectangular area unchanged, and the other pixels are set to zero.

Step 3: Apply inverse Radon transform to enhanced image, and the recovered image is gotten.

Fig.5 shows the recovered image, it can be seen that noises from voltaic arc and molten steel spray are almost eliminated, and little recovering noises are generated, while the laser stripe and weld seam groove are kept well. 


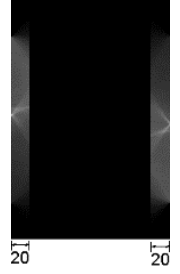

(a) Enhancement

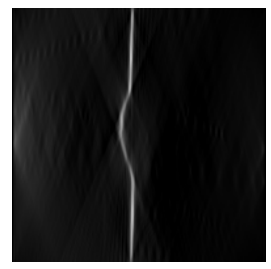

(b) Image recovery

Fig.5 Image enhancement in Radon transform domain and recovery

\section{Improved fast FCM clustering image segmentation algorithm}

After the elimination of image noises, it is necessary to carry out image segmentation so that the laser stripe can be separated from the background of the image. At present, FCM (fuzzy C-means) clustering algorithm is widely used in image segmentation. Suppose $X=\left\{x_{1}, x_{2}, x_{3}, \ldots, x_{n}\right\}$ represents data sets, $O=\left\{o_{1}, o_{2}, o_{3}, \ldots, o_{c}\right\}(2 \leq c \leq n)$ is the cluster centers, FCM algorithm is aimed to classify $X$ into $c$ clusters. Assume $u_{i j}$ is the membership grade of sample $j$ belonging to center $i$, so

$$
u_{i j} \in[0,1], \quad \sum_{i=1}^{c} u_{i j}=1
$$

The objective function of FCM is

$$
J=\sum_{j=1}^{n} \sum_{i=1}^{c} u_{i j}^{m} d_{i j}^{2}
$$

Where $d_{i j}=\left\|x_{j}-o_{i}\right\|$ is Euler distance between sample $x_{j}$ and center $o_{i}, m \geq 1$ is fuzzy coefficient. FCM algorithm is about finding minimum value of objective function $J$ under constrain of formula (5).

As one image always have many pixels, for a $256 \times 256$ size image, there are 65536 pixels, much time will be cost if FCM is directly applied for segmentation. In this paper, an improved fast FCM algorithm based on image histogram is used for image segmentation. Assume the number of image's gray level is $L$, the input vector in improved FCM algorithm is defined as

$$
x_{l}=\left[\frac{l}{L-1}, f(l)\right]^{T}
$$

Where $l$ is gray level, $n_{l}$ is the total number of pixels whose gray levels are $l, N$ is the total number of pixels in the image, $p_{l}=p(l)=n_{l} / N$ represents the appearing probability of gray level $L, f(l)=\sum_{j=0}^{l} p(j)$ represents the appearing probability of gray level range $[0, l]$. Therefore, the objective function of improved FCM is modified as

$$
J=\sum_{l=0}^{L-1} \sum_{i=1}^{c} u_{i l}^{m} p_{l} d_{i l}^{2}
$$

As an image generally has 256 gray levels, therefore the FCM algorithm based on histogram is much faster. As shown in Fig.6, apply the improved fast FCM algorithm to Fig.5b for segmentation, it can be seen that the laser stripe is almost separated from the image background, only a few noises are improperly classified, it is easy to get the groove width, center, turning point A and B, etc. 


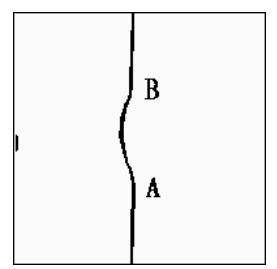

Fig.6 Laser stripe image after improved FCM segmentation

The above steps are the whole image processing procedure. For a $192 \times 192$ size image, the cost time is about $0.21 \mathrm{~s}$ in the $3.2 \mathrm{GHZ}$ CPU computer. Therefore, these image processing ways can meet the requirement of real-time seam tracking and control. Many experiments results show that these methods are robust and have good precision.

\section{Conclusion}

In the procedure of weld seam tracking with laser vision, the weld seam image is mainly polluted by the noises from voltaic arc and molten steel spray. Radon transform can be applied to the weld seam image, the information in Radon transform domain corresponding to the noises in weld seam image can be eliminated easily, while the information in Radon transform domain corresponding to the laser stripe can be kept well. Then, inverse Radon transform for image recovery is used, there are little noises in the recovered image. Good results can be gotten when the improved fast FCM clustering segmentation is used for laser stripe extraction.

\section{Acknowledgments}

This work was sponsored by the Science and Technology Fund Project of Zhaoqing University(Project No. 201758).

\section{References}

1. X.W. Liu, G.R. Wang, Y. H, Shi, Image processing in welding seam tracking based on singlestripe laser, Transactions of the China Welding Institution. Vol.27, No.6, June 2006, pp.2528,32. (in chinese)

2. K.F. Deng, et al, Laser technology and application. Changsha:Defence Science and technology press, 2002, pp.275. (in chinese)C.Jarlskog, CP Violation (World Scientific, Singapore, 1988).

3. Q.Q. Ruan, Digital image processing. Beijing:Electron industry press, 2013.(in chinese)

4. Hung M C, Yang D L, An efficient fuzzy c-means clustering algoritm, Pcoceedings of IEEE International Conference on Data Mining, SanJose, 2001, pp.225-232.

5. Tolias Y A, Panas S M, Image segmentation by a fuzzy clustering algorithm using adapitive spatially constrained functions, IEEE Trans Syst, Man, Cybernet Part, Vol.28, No.3, 1998, pp.359-369. 